\title{
To Compare Efficacy of Nishkatakadi Kwath with Metformin in Madhumeha with Special
} Refrence to Diabetes Mellitus ii

\section{Bhairav B. Tawshikar ${ }^{1 *}$,Shrikant G. Deshmukh ${ }^{2}$, Yashashree B. Kulkarni ${ }^{3}$}

${ }^{1}$ Kayachikitsa, Ashvin Rural Ayurved College, Manchihill, Aurangabad,India

${ }^{2}$ Kayachikitsa Deptt., C.S.M.S.S. Ayurved College, Aurangabad, India

${ }^{3}$ PG Scholar, Rognidan Deptt., C.S.M.S.S. Ayurved College, Aurangabad, India

*Corresponding Author: Bhairav B. Tawshikar, Kayachikitsa, Ashvin Rural Ayurved College, Manchihill, Aurangabad, India

Received date: December 15, 2019; Accepted date: January 06, 2019; Published date: January 14, 2020

Citation: Bhairav B. Tawshikar, Shrikant G. Deshmukh, Yashashree B. Kulkarni (2020). To Compare Efficacy of Nishkatakadi Kwath with Metformin in Madhumeha With Special Refrence to Diabetes Mellitus ii 3(1); Doi: 10.31579/ 2641-8975 /016

Copyright: (92020 Bhairav B. Tawshikar, This is an open-access article distributed under the terms of The Creative Commons. Attribution License, which permits unrestricted use, distribution, and reproduction in any medium, provided the original author and source are credited.

\begin{abstract}
Madhumeha is a disease known since ancient times to the mankind, its upsurge is quiet alarming. On the basis of its symptomatology Madhumeha can be correlated to the features of Diabetes mellitus.

Diabetes mellitus is a metabolic disorder of carbohydrate, fat, \& protein characterized by hyperglycemia with or without glycosuria. It is associated with long-term potentially catastrophic effects on almost all systems of the body.

Ayurveda can provide better management for Madhumeha without hazardous side effects. In Ayurveda, Madhumeha has been described as one among one of the 20 types of Prameha and is a sub-type of Vatika Prameha. In the former type the patients are usually asthenic can be correlated with Type I DM and in the latter type patients are obese and can be equated with Type II DM. Nishakatakadi Kwath has been mentioned in Sahasrayoga under Kashaya Prakaran for Prameha Chikitsa, Nishakatakadi Kwath is being used widely for the treatment of Madhumeha in Kerala. That is why Nishakatakadi Kwath has been selected for the present study.

In this study to study various clinical symptoms of madhumeha with special reference to Diabetes Mellitus II. To compare efficacy of Nishkatakadi Kwath with Metformin inthe management of Madhumeha,

To observe any possible adverse effect of Nishkatakadi kwath if reported during the study. In the present study Trial and Control groups comprising 30 patients each of Madhumeha (Diabetes Mellitus type II) were treated with Nishkatakadi kwath with tab metformin and Tab Metformin alone respectively. This study shows that both the drugs NISHKATAKADI KWATH with metformin are more effective than METFORMIN in Madhumeha to reduce symptoms and patients score.
\end{abstract}

\section{Key words- Madhumeha, Nishkatkadi Kwath, Diabetic Mellitus}

\section{Introduction}

On the basis of its symptoms of Madhumeha can be correlated to the features of Diabetes mellitus.

Diabetes mellitus is a metabolic disorder of carbohydrate, fat, \&protein characterized by hyperglycemia with or without glycosuria. It is associated with long-term potentially catastrophic effects on almost all systems of the body. These can manifest as minor annoyances at first but then insidiously destroy the cellular components of a given body part, organ, or entire system. Diabetes Mellitus is aggressively progressive and the prognosis is poorunless definite measures are taken to control the disease. At the present time there is no known cure for DM and even with proper medical management; prognosis may still be poor due to irreversible major impairments or severe disabilities. It is most often treated with diet and exercise, typically in conjunction with oral hyperglycemic drugs (OHD). Ayurveda can provide better management for Madhumeha without hazardous side effects. In Ayurveda, Madhumeha has been described as one among one of the 20 types of Prameha and is a sub-type of Vatika Prameha ${ }^{1}$

Nishakatakadi Kwath has been mentioned in Sahasrayoga under Kashaya Prakaran for Prameha
Chikitsa ${ }^{2}$. Nishakatakadi Kwath is being used widely for the treatment of Madhumeha in Kerala. That is why Nishakatakadi Kwath has been selected for the present study.

\section{Aim and Objectives:}

1) To study various clinical symptoms of madhumeha with special refrence to Diabetes Mellitus II.

2) To compare efficacy of Nishkatakadi kwath with Metformine in the management of Madhumeha.

3) To observe any possible adverse effect of Nishkatakadi Kwath if reported during the study.

4) To study in details about Madhumeha according to available ayurvedic literature.

5) To study in details about Diabetic Mellitus according to Modern science.

\section{Methods and Materials:}

Patients having chief complains of Prameha having features like Prabhutmutrata, Pipasa, Alasya, Angamard, etc. Diabetes Mellitus was selected randomly on the OPD \& I.P.D basis of Kaychikitsa

Department of our college.60 patients was divided by Randomisation, subjects in group A and subjects in group B. 
Group A- Nishkatadi kwath + Tab. Metformin3

DOSE $20 \mathrm{ml}-\mathrm{BD}+500 \mathrm{mg}-\mathrm{BD}$

ANUPAN- Water, SEVAN KAL - Adhobhakt(After meal) ROUTE OF ADMINISTRATION - Oral ,DURATION OF TREATMENT 45 Days

FOLLOW UP - After every 15 days

Group B(control group) METFORMIN+ NIDANPARIVARJAN

Metformin - 500 mg-BD Anupan - Water, After meal , Oral -45 Days, Follow Up - After every 15 Days.

\section{Selection Criteria:}

InclusionCriteria:

Patients representing signs and symptoms of Madhumeha.

Patient age group with 16-60 yr.

Patient with BSL Fasting 110-200mg/dl and Post Prandial160-350mg/dl.

Patients willing to participate in the study will be selected explaining them details about study.

\section{Exclusion Criteria -}

Patients belonging to age group below 16 and above 60 years.

Patient with any illness or Prameha associated with other systemic diseases.

Fasting BSL $<110 \mathrm{mg} / \mathrm{dl}$ and Post Prandial $<160 \mathrm{mg} / \mathrm{dl}$.

KnowncaseofIDDM

Objective Criteria 1)BSL 2) Urine Routine 3) HbA1c

DURATION: 45Days., FOLLOW UP: D0, D15, D30, D45.

PLACE OF WORK: Ashvin Rural Ayurved College, Manchi

Hill,Tal.Sangamner, Dist. Ahmednagar.

Preparation of Drug:The drug was prepared in the dept. of Rasa Shashtra and Bhaishajya Kalpana, Ashvin Rural Ayurved College, Manchihill, Tal.Sangamner, Dist. Ahmednagar,

Contents of Nishkatakadi kwath.

1. Nisha ${ }^{4}$ (Haridra)- Latin name: Curcuma longa Family: Zingiberaceae

The antioxidant properties of curcuma powder are probably due to the phenolic character of curcumin. (The wealth of India)

Therapeutic uses: Prameha, Shitapitta, Pandu, Kustha, Kandu 2. AMALKI ${ }^{5}$ - Latin name: Embilica officinalis

Family: Euphorbiaceae

Part Used: Fruit

Chemical Constituents: Fruit contains gallic acid, tannic acid, sugars, albumin, cellulose and minerals. Therapeutic uses: Prameha, Hridroga, Yakritroga, Kustha, Raktapitta. Pharmacological Actions: Fresh fruit is diuretic and laxative.

3. KATAKA ${ }^{\mathbf{6}}$ : Latin name: Strychnos potatorum Family: Loganiaceae

Part Used: Seed Chemical Constituents:Seeds contain no strychnine but brucine is present.
4. PARANTI ${ }^{7}$ : Latin name: Ixora coccinea Family: Rubiaceae, Part Used: Root, Chemical Constituents: Root contains acrid aromatic oil, tannin, fatty acids, and a white crystalline substance. The root bark contains octadecadienoic acid, mannitol and myristic acid.,Pharmacological Actions:Roots are reported to possess sedative and stomachic properties, stimulate gastric secretions \& bile and also possess astringent and antiseptic properties

5. LODHRA ${ }^{\mathbf{8}}$ : Latin name: Symplocos racemosa Family: Symplocaceae

Part Used: Bark, Chemical Constituents: Bark contains following alkaloids:-0.24\%Loturine, $0.02 \%$ Colloturine, $0.06 \%$ Loturidine and Quinovin

6. USHIRA9: Latin name: Vetiveria zizanioidis Family: Graminae

Part Used: Root Chemical Constituents: A volatile essential oil, resin, colouring matter, a free acid, a salt of lime, oxide of iron and woody matters.

Actions (Karma): $\quad$ Twakdoshhar, Varnya,Dahaprashaman, Trishnanigrahana, Stambhana, Swedapanyana, Raktaprasadana.

BSL Fasting and Post meal - Group A - As value of $\mathrm{p}$ is far less than 0.05 extremely significant differences was observed between mean of BT and AT score in BSL fasting parameter. Hence it is concluded that Nishkatakadi kwath with metformin is highly effective to reduce BSL fasting in Madhumeha (DM II)

Group B As value of $\mathrm{p}$ is far less than 0.05 extremely significant differences was observed between mean of BT and AT score in BSL fasting parameter. Hence it is concluded that Tab Metformin is highly effective to reduce BSL fasting in Madhumeha (DM II).

HBA1C - Group A - As value of $\mathrm{p}$ is far less than 0.05 extremely significant differences was observed between mean of BT and AT score in $\mathrm{HbA1C}$ parameter. Hence it is concluded that Nishkatakadi Kwath along with Metformin is highly effective to reduce HbA1C in Madhumeha (DM II)

Group B- As value of $\mathrm{p}$ is far less than 0.05 extremely significant differences was observed between mean of BT and AT score in HbA1C parameter. Hence it is concluded that Tab Metformin is highly effective to reduce $\mathrm{HbA} 1 \mathrm{C}$ in Madhumeha (DM II).

Hence it is concluded that Nishkatakadi kwath along with metformin is highly effective to reduce USL (random) in Madhumeha (DM II).

USL - Group A- As value of $p$ is far less than 0.05 extremely significant differences was observed between mean of BT and AT score in USL (random) parameter Hence it is concluded that Nishkatakadi kwath along with metformin is highly effective to reduce USL (random) in Madhumeha (DM II).

Group B- As value of $\mathrm{p}$ is far less than 0.05 extremely significant differences was observed between mean of BT and AT score in USL (random) parameter. Hence it is concluded that Tab Metformin is highly effective to reduce USL (random) in Madhumeha (DM II).

Discussion:In the case of symptoms Prabhutamutrata, Naktmutrata, Pipasavrudhi, Kshudhavrudhi, Hastpadtal daha, Swedapravrutti and Anutsaha the test has shown insignificant difference between mean differences of Group A and Group B. H1 is accepted and $\mathbf{H}_{\mathbf{0}}$ is rejected here. It was hence concluded that Nishkatakadi kwath along with tab metformin is effective than Tab Metformin alone to reduce Prabhutamutrata, Naktmutrata, Pipasavrudhi, Kshudhavrudhi, Hastpadtal daha, Swedapravrutti and Anutsaha in Madhumeha (DM II).

Decrease in Objective parameters before and after treatment

Average decrease in BSL (F) in Group A is $\mathbf{6 9 . 0 3 ~ m g / d l ~ w h i l e ~ i n ~ G r o u p ~}$ $B$ is 67.9. Average decrease in BSL (PP) in Group A is $84.53 \mathrm{mg} / \mathrm{dl}$ while 
in Group B is 84.17. Average decrease in USL (R) in Group A is 2.1 while in Group B is 1.87. Average decrease in HbA1C in Group A is 3.24 while in Group B is $\mathbf{2 . 3}$.

Conclusion:To sum up, it can be said that the present study shows significant remission in Signs and symptoms of illness Madhumeha with related to type 11 Diabetes Mellitus confirmed that definite reduction in blood sugar levels. Therefore it is imperative that the combination of Nishkatakadi kwath and tab metformin is helps in management of the disease.

Summary:The research study was carried out under following headings:-

Observations and Results:

This part consists of the demographic data of the patients, comparison of the subjective and objective parameters before and after the treatment and the overall response to the treatment. The symptoms likes' prabhut mutrata, naktmutrata, pipasavrudhi, kshudhavrudhi, hastpadtal daha, swedatipravrutti and anutsaha found excellent significant effect in both groups.

Statistical Analysis: For statistical analysis, Wilcoxon signed rank test and Mann Whitney's U test is used for subjective criteria.

Discussion: Madhumeha is mainly found in Vat-Pittaj prakruti patients. This shows that the predominance of Vata, Pittadoshasin Prameha\& males are more affected than females. There is significant improvement found in symptoms of Madhumeha patient safer giving both the drugs.

\section{Master Chart - Group A}

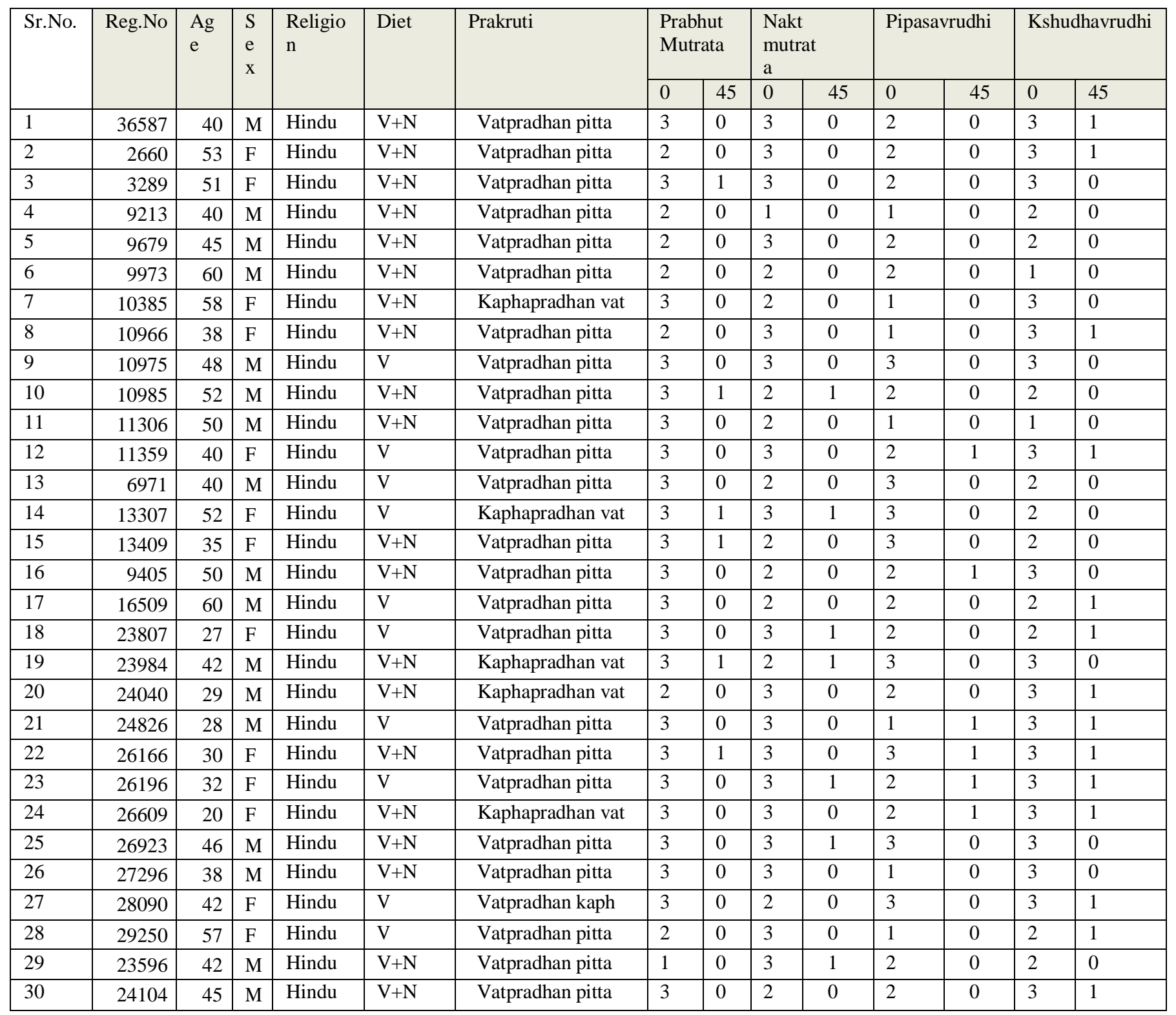




\begin{tabular}{|c|c|c|c|c|c|c|c|c|c|c|c|c|c|}
\hline \multirow{2}{*}{\multicolumn{2}{|c|}{$\begin{array}{l}\text { Hastpa } \\
\text { Dtal } \\
\text { daha }\end{array}$}} & \multirow{2}{*}{\multicolumn{2}{|c|}{$\begin{array}{l}\text { Swedati } \\
\text { pravritti }\end{array}$}} & \multirow{2}{*}{\multicolumn{2}{|c|}{$\begin{array}{l}\text { Anutsa } \\
\text { ha }\end{array}$}} & \multicolumn{4}{|l|}{ BSL } & \multirow{3}{*}{\multicolumn{2}{|c|}{$\begin{array}{l}\text { Urine } \\
\text { Examinati } \\
\text { on }\end{array}$}} & \multirow{3}{*}{\multicolumn{2}{|c|}{$\mathrm{Hb} \mathrm{A} 1 \mathrm{C}$}} \\
\hline & & & & & & \multirow{2}{*}{\multicolumn{2}{|c|}{$\begin{array}{l}1 \mathrm{st} \\
\mathrm{BT} \\
\end{array}$}} & \multirow{2}{*}{\multicolumn{2}{|c|}{$\begin{array}{l}45 \text { th } \\
\text { AT } \\
\end{array}$}} & & & & \\
\hline 0 & 45 & 0 & 45 & 0 & 45 & & & & & & & & \\
\hline 2 & 0 & 2 & 0 & 3 & 1 & $F$ & $\mathrm{PP}$ & $F$ & $\mathrm{PP}$ & $1 \mathrm{st}$ & $\begin{array}{l}45 \\
\text { th }\end{array}$ & BT & $\mathrm{AT}$ \\
\hline 2 & 1 & 3 & 1 & 2 & 0 & 180 & 240 & 98 & 140 & +++ & $\mathrm{Ab}$ & 11 & 7.8 \\
\hline 2 & 1 & 3 & 0 & 2 & 0 & 170 & 250 & 110 & 170 & ++ & $\mathrm{Ab}$ & 10 & 6.7 \\
\hline 1 & 0 & 2 & 0 & 1 & 0 & 148 & 248 & 88 & 110 & ++ & $\mathrm{Ab}$ & 11 & 8.2 \\
\hline 2 & 0 & 1 & 0 & 2 & 0 & 136 & 200 & 86 & 135 & +++ & $\mathrm{Ab}$ & 10 & 6.4 \\
\hline 2 & 0 & 1 & 0 & 1 & 0 & 180 & 244 & 121 & 180 & ++ & $\mathrm{Ab}$ & 9.8 & 7 \\
\hline 1 & 0 & 2 & 1 & 2 & 0 & 156 & 255 & 75 & 135 & +++ & $\mathrm{Ab}$ & 9.5 & 6.5 \\
\hline 1 & 0 & 2 & 0 & 3 & 0 & 126 & 260 & 90 & 153 & +++ & $\mathrm{Ab}$ & 9.1 & 6.6 \\
\hline 2 & 1 & 2 & 1 & 2 & 0 & 167 & 229 & 110 & 139 & ++ & $\mathrm{Ab}$ & 10 & 6 \\
\hline 1 & 0 & 1 & 0 & 3 & 0 & 170 & 250 & 93 & 150 & ++ & $\mathrm{Ab}$ & 9.8 & 7.1 \\
\hline 1 & 0 & 2 & 0 & 1 & 0 & 178 & 253 & 90 & 175 & ++ & $\mathrm{Ab}$ & 9.2 & 7.8 \\
\hline 1 & 0 & 2 & 0 & 3 & 1 & 178 & 260 & 101 & 157 & +++ & + & 11 & 7 \\
\hline 1 & 0 & 2 & 0 & 2 & 0 & 172 & 252 & 92 & 122 & ++ & $\mathrm{Ab}$ & 9.4 & 6.7 \\
\hline 2 & 0 & 2 & 0 & 3 & 1 & 175 & 238 & 70 & 145 & ++ & $\mathrm{Ab}$ & 10 & 6.2 \\
\hline 3 & 0 & 2 & 0 & 3 & 1 & 177 & 229 & 91 & 147 & +++ & + & 9.5 & 6.6 \\
\hline 2 & 0 & 3 & 0 & 3 & 0 & 173 & 228 & 89 & 162 & ++ & + & 9.8 & 6.3 \\
\hline 2 & 0 & 2 & 0 & 3 & 1 & 140 & 214 & 98 & 160 & ++ & + & 7.6 & 6 \\
\hline 2 & 0 & 1 & 0 & 3 & 1 & 165 & 218 & 71 & 145 & +++ & $\mathrm{Ab}$ & 10 & 6.2 \\
\hline 2 & 0 & 1 & 0 & 3 & 1 & 170 & 200 & 121 & 185 & +++ & + & 11 & 6 \\
\hline 2 & 0 & 1 & 0 & 2 & 0 & 168 & 258 & 145 & 190 & +++ & + & 10 & 6.3 \\
\hline 1 & 0 & 1 & 0 & 2 & 0 & 168 & 208 & 98 & 140 & ++ & $\mathrm{Ab}$ & 11 & 7 \\
\hline 2 & 1 & 2 & 1 & 2 & 0 & 166 & 218 & 89 & 150 & +++ & $\mathrm{Ab}$ & 11 & 6.5 \\
\hline 2 & 0 & 1 & 0 & 3 & 0 & 180 & 230 & 78 & 149 & + & $\mathrm{Ab}$ & 11 & 6 \\
\hline 2 & 1 & 1 & 0 & 2 & 0 & 126 & 201 & 87 & 116 & ++ & $\mathrm{Ab}$ & 9.6 & 6.1 \\
\hline 1 & 0 & 2 & 0 & 3 & 0 & 175 & 226 & 100 & 139 & ++ & $\mathrm{Ab}$ & 12 & 8 \\
\hline 1 & 0 & 2 & 0 & 2 & 0 & 169 & 246 & 82 & 160 & +++ & + & 10 & 6.1 \\
\hline 2 & 1 & 1 & 0 & 2 & 0 & 143 & 240 & 124 & 185 & ++ & $\mathrm{Ab}$ & 10 & 8.2 \\
\hline 3 & 0 & 2 & 1 & 2 & 0 & 143 & 250 & 76 & 132 & +++ & $\mathrm{Ab}$ & 12 & 7.1 \\
\hline 3 & 1 & 2 & 1 & 3 & 1 & 131 & 236 & 101 & 140 & ++ & + & 8 & 6.2 \\
\hline 2 & 0 & 2 & 0 & 1 & 0 & 158 & 213 & 71 & 138 & +++ & $\mathrm{Ab}$ & 8.8 & 7.4 \\
\hline & & & & & & 175 & 238 & 81 & 158 & +++ & + & 9.6 & 6.4 \\
\hline
\end{tabular}

\section{Master Chart - Group B}

\begin{tabular}{|c|c|c|c|c|c|c|c|c|c|c|c|c|c|c|c|c|}
\hline \multirow[t]{2}{*}{$\begin{array}{l}\text { Sr. } \\
\text { No. }\end{array}$} & \multirow[t]{2}{*}{ Reg.No } & \multirow[t]{2}{*}{ Age } & \multirow[t]{2}{*}{ Sex } & \multirow[t]{2}{*}{ Religion } & \multirow[t]{2}{*}{ Diet } & \multirow[t]{2}{*}{ Prakruti } & \multicolumn{2}{|c|}{$\begin{array}{l}\text { Prabhut } \\
\text { Mutrata }\end{array}$} & \multicolumn{2}{|c|}{$\begin{array}{l}\text { Nakt } \\
\text { mutrata }\end{array}$} & \multicolumn{2}{|c|}{$\begin{array}{l}\text { Pipasa } \\
\text { vrudhi }\end{array}$} & \multicolumn{2}{|c|}{$\begin{array}{c}\text { Kshud } \\
\text { havrud } \\
\text { hi }\end{array}$} & \multicolumn{2}{|c|}{$\begin{array}{l}\text { Hastp } \\
\text { adtal } \\
\text { daha }\end{array}$} \\
\hline & & & & & & & 0 & 45 & 0 & 45 & $\overline{0}$ & 45 & 0 & 45 & 0 & 45 \\
\hline 1 & 2563 & 53 & $\mathrm{~F}$ & Hindu & $\mathrm{V}+\mathrm{N}$ & Vatpradhan pitta & 3 & 0 & 2 & 1 & 3 & 1 & 3 & 0 & 1 & 0 \\
\hline 2 & 2711 & 55 & M & Hindu & $\mathrm{V}+\mathrm{N}$ & Kaphapradhan Vat & 2 & 1 & 2 & 1 & 1 & 0 & 2 & 1 & 2 & 0 \\
\hline 3 & 7063 & 25 & M & Hindu & $\mathrm{V}+\mathrm{N}$ & Vatpradhan Kaph & 2 & 1 & 2 & 1 & 3 & 0 & 3 & 0 & 2 & 0 \\
\hline 4 & 9404 & 26 & M & Hindu & $\mathrm{V}+\mathrm{N}$ & Vatpradhan pitta & 2 & 0 & 2 & 0 & 2 & 0 & 2 & 0 & 1 & 0 \\
\hline 5 & 10362 & 50 & $\mathrm{M}$ & Hindu & $\mathrm{V}+\mathrm{N}$ & Vatpradhan pitta & 3 & 0 & 3 & 1 & 3 & 1 & 3 & 0 & 1 & 0 \\
\hline 6 & 10722 & 30 & M & Hindu & V & Vatpradhan pitta & 3 & 0 & 2 & 1 & 1 & 0 & 1 & 0 & 1 & 0 \\
\hline
\end{tabular}




\begin{tabular}{|c|c|c|c|c|c|c|c|c|c|c|c|c|c|c|c|c|}
\hline 7 & 10971 & 54 & M & Hindu & $\mathrm{V}+\mathrm{N}$ & Vatpradhan pitta & 1 & 0 & 3 & 0 & 3 & 0 & 3 & 0 & 1 & 0 \\
\hline 8 & 10976 & 37 & $\mathrm{~F}$ & Hindu & $\mathrm{V}+\mathrm{N}$ & Vatpradhan pitta & 1 & 0 & 2 & 1 & 3 & 0 & 3 & 1 & 1 & 0 \\
\hline 9 & 10980 & 39 & $\mathrm{M}$ & Hindu & $\mathrm{V}+\mathrm{N}$ & Vatpradhan pitta & 3 & 1 & 2 & 1 & 3 & 1 & 3 & 1 & 2 & 1 \\
\hline 10 & 11354 & 35 & M & Hindu & $\mathrm{V}+\mathrm{N}$ & Kaphapradhan Vat & 2 & 0 & 2 & 0 & 3 & 1 & 3 & 0 & 1 & 0 \\
\hline 11 & 6980 & 50 & $\mathrm{~F}$ & Hindu & $\mathrm{V}+\mathrm{N}$ & Kaphapradhan Vat & 2 & 0 & 3 & 0 & 3 & 1 & 3 & 0 & 1 & 0 \\
\hline 12 & 11857 & 30 & $\mathrm{~F}$ & Hindu & $\mathrm{V}+\mathrm{N}$ & Vatpradhan pitta & 2 & 1 & 3 & 0 & 3 & 1 & 3 & 1 & 1 & 0 \\
\hline 13 & 13385 & 54 & $\mathrm{M}$ & Hindu & $\mathrm{V}+\mathrm{N}$ & Pittapradhan Vat & 3 & 0 & 3 & 0 & 3 & 0 & 3 & $\overline{0}$ & 1 & $\overline{0}$ \\
\hline 14 & 14157 & 58 & M & Hindu & $\mathrm{V}+\mathrm{N}$ & Vatpradhan pitta & 2 & 0 & 3 & 1 & 3 & 1 & 3 & 1 & 2 & 1 \\
\hline 15 & 10985 & 52 & $\mathrm{M}$ & Hindu & $\mathrm{V}+\mathrm{N}$ & Vatpradhan pitta & 3 & 0 & 3 & 0 & 3 & 1 & 2 & 0 & 1 & 0 \\
\hline 16 & 23811 & 50 & $\mathrm{M}$ & Hindu & $\mathrm{V}$ & Kaphapradhan Vat & 2 & 0 & 2 & 0 & 3 & 1 & 3 & 1 & 2 & 0 \\
\hline 17 & 24030 & 55 & $\mathrm{M}$ & Hindu & $\mathrm{V}$ & Kaphapradhan Vat & 3 & 0 & 3 & 0 & 3 & 1 & 3 & 0 & 3 & 0 \\
\hline 18 & 24510 & 42 & $\mathrm{M}$ & Hindu & $\mathrm{V}+\mathrm{N}$ & Kaphapradhan Vat & 3 & 0 & 2 & $\overline{0}$ & 3 & 1 & 2 & $\overline{0}$ & 3 & 0 \\
\hline 19 & 26122 & 34 & $\mathrm{M}$ & Hindu & $\mathrm{V}$ & Vatpradhan pitta & 3 & 1 & 2 & 0 & 3 & 1 & 2 & 0 & 3 & 0 \\
\hline 20 & 26191 & 37 & $\mathrm{M}$ & Hindu & $\mathrm{V}+\mathrm{N}$ & Vatpradhan pitta & 3 & 1 & 2 & 0 & 1 & 0 & 3 & 0 & 1 & 0 \\
\hline 21 & 26298 & 32 & $\mathrm{M}$ & Hindu & $\mathrm{V}+\mathrm{N}$ & Pittapradhan Vat & 3 & 1 & 3 & 1 & 3 & 0 & 3 & 1 & 1 & 0 \\
\hline 22 & 26715 & 32 & $\mathrm{M}$ & Hindu & $\mathrm{V}+\mathrm{N}$ & Kaphapradhan Vat & 2 & 1 & 2 & 0 & 3 & 1 & 3 & 0 & 1 & 0 \\
\hline 23 & 27112 & 29 & $\mathrm{~F}$ & Hindu & $\mathrm{V}$ & Kaphapradhan Vat & 2 & 1 & 2 & $\overline{0}$ & 3 & 0 & 3 & $\overline{0}$ & 2 & $\overline{0}$ \\
\hline 24 & 28085 & 40 & $\mathrm{M}$ & Hindu & $\mathrm{V}+\mathrm{N}$ & Vatpradhan pitta & 3 & 1 & 3 & 0 & 3 & 1 & 3 & 0 & 3 & 1 \\
\hline 25 & 28995 & 60 & $\mathrm{M}$ & Hindu & $\mathrm{V}+\mathrm{N}$ & Vatpradhan pitta & 3 & 1 & 3 & 1 & 2 & 0 & 3 & 0 & 1 & 0 \\
\hline 26 & 29360 & 60 & $\mathrm{~F}$ & Hindu & $\mathrm{V}+\mathrm{N}$ & Vatpradhan pitta & 1 & 0 & 2 & 1 & 2 & 0 & 2 & 1 & 1 & 0 \\
\hline 27 & 23597 & 34 & $\mathrm{~F}$ & Hindu & $\mathrm{V}$ & Vatpradhan pitta & 3 & 1 & 2 & 0 & 3 & 0 & 3 & 0 & 2 & 0 \\
\hline 28 & 23970 & 27 & $\mathrm{M}$ & Hindu & $\mathrm{V}+\mathrm{N}$ & Vatpradhan pitta & 2 & 1 & 2 & 0 & 2 & 0 & 2 & 1 & 2 & 1 \\
\hline 29 & 13967 & 57 & $\mathrm{M}$ & Hindu & $\mathrm{V}$ & Vatpradhan pitta & 3 & 0 & 2 & 0 & 2 & 0 & 2 & 1 & 2 & 0 \\
\hline 30 & 34105 & 27 & $\mathrm{~F}$ & Hindu & $\mathrm{V}+\mathrm{N}$ & Vatpradhan pitta & 2 & 1 & 3 & 0 & 3 & 0 & 2 & 0 & 3 & 0 \\
\hline
\end{tabular}

\begin{tabular}{|c|c|c|c|c|c|c|c|c|c|c|c|}
\hline \multicolumn{2}{|c|}{ Swedpravrutti } & \multirow{2}{*}{\multicolumn{2}{|c|}{ Anustha }} & \multicolumn{4}{|c|}{ BSL } & \multirow{3}{*}{\multicolumn{2}{|c|}{$\begin{array}{c}\text { Urine } \\
\text { Examination }\end{array}$}} & \multirow{3}{*}{\multicolumn{2}{|c|}{$\mathrm{Hb}$ A1C }} \\
\hline \multirow{3}{*}{$\begin{array}{l}0 \\
2\end{array}$} & \multirow{3}{*}{$\begin{array}{c}45 \\
1\end{array}$} & & & \multirow{2}{*}{\multicolumn{2}{|c|}{$\begin{array}{l}1 \\
\text { st }\end{array}$}} & \multirow{2}{*}{\multicolumn{2}{|c|}{$\begin{array}{c}45 \text { th } \\
\mathrm{A} \\
\mathrm{T}\end{array}$}} & & & & \\
\hline & & 0 & 45 & & & & & & & & \\
\hline & & 2 & 0 & $\mathrm{~F}$ & PP & $\mathrm{F}$ & $\mathrm{PP}$ & $1 \mathrm{st}$ & 45 th & $\begin{array}{l}\mathrm{B} \\
\mathrm{T}\end{array}$ & AT \\
\hline 2 & 1 & 2 & 1 & 168 & 223 & 99 & 170 & +++ & + & 10 & 7.2 \\
\hline 1 & 1 & 2 & 1 & 176 & 200 & 89 & 148 & ++ & Trace & 10 & 7 \\
\hline 3 & 0 & 2 & 0 & 178 & 258 & 100 & 158 & ++ & Trace & 8 & 6 \\
\hline 3 & 1 & 3 & 0 & 169 & 256 & 101 & 135 & ++ & + & 8.5 & 6 \\
\hline 2 & 1 & 2 & 1 & 160 & 200 & 88 & 154 & +++ & Trace & 10 & 7.5 \\
\hline 3 & 0 & 3 & 1 & 180 & 222 & 74 & 136 & ++ & + & 8.3 & 6.1 \\
\hline 2 & 0 & 3 & 0 & 172 & 226 & 75 & 143 & +++ & Trace & 7.9 & 6 \\
\hline 3 & 0 & 3 & 1 & 140 & 248 & 79 & 145 & +++ & $\mathrm{Ab}$ & 11 & 7.2 \\
\hline 3 & 1 & 2 & 0 & 150 & 250 & 98 & 148 & ++ & $\mathrm{Ab}$ & 7.1 & 6.2 \\
\hline 2 & 0 & 3 & 1 & 165 & 243 & 110 & 140 & + & $\mathrm{Ab}$ & 10 & 7.2 \\
\hline 3 & 0 & 2 & 0 & 152 & 253 & 78 & 110 & ++ & $\mathrm{Ab}$ & 9.2 & 6.9 \\
\hline 3 & 1 & 3 & 1 & 166 & 201 & 70 & 135 & +++ & Trace & 10 & 6.1 \\
\hline 2 & 1 & 3 & 0 & 178 & 256 & 118 & 160 & ++ & $\mathrm{Ab}$ & 9 & 6.2 \\
\hline 3 & 1 & 2 & 1 & 166 & 258 & 110 & 147 & ++ & + & 8.6 & 6.3 \\
\hline 2 & 0 & 2 & 0 & 173 & 255 & 97 & 171 & ++ & + & 7.6 & 6.7 \\
\hline 2 & 0 & 2 & 0 & 174 & 259 & 105 & 145 & +++ & $\mathrm{Ab}$ & 8 & 6 \\
\hline
\end{tabular}




\begin{tabular}{|c|c|c|c|c|c|c|c|c|c|c|c|}
\hline 2 & 0 & 3 & 0 & 177 & 247 & 101 & 153 & ++ & + & 8.2 & 6 \\
\hline 3 & 0 & 2 & 1 & 145 & 221 & 95 & 170 & +++ & Trace & 10 & 7.8 \\
\hline 2 & 1 & 3 & 1 & 156 & 210 & 92 & 154 & +++ & Ab & 11 & 10 \\
\hline 2 & 1 & 3 & 1 & 170 & 235 & 94 & 210 & +++ & + & 11 & 10 \\
\hline 2 & 1 & 2 & 1 & 168 & 226 & 121 & 140 & +++ & Ab & 8.5 & 7 \\
\hline 3 & 1 & 2 & 1 & 158 & 236 & 81 & 145 & +++ & Ab & 9.2 & 6 \\
\hline 3 & 0 & 3 & 0 & 148 & 245 & 98 & 165 & ++ & Ab & 6.3 & 6 \\
\hline 2 & 0 & 3 & 0 & 139 & 258 & 89 & 170 & + & Ab & 11 & 7 \\
\hline 2 & 0 & 3 & 0 & 148 & 210 & 92 & 149 & +++ & Trace & 9.2 & 6.5 \\
\hline 1 & 0 & 1 & 0 & 139 & 250 & 81 & 142 & +++ & + & 10 & 7.8 \\
\hline 2 & 0 & 2 & 0 & 153 & 245 & 79 & 129 & ++ & Ab & 6.3 & 6 \\
\hline 3 & 0 & 2 & 0 & 168 & 220 & 80 & 161 & +++ & + & 9.5 & 6.2 \\
\hline 3 & 1 & 3 & 0 & 138 & 223 & 84 & 143 & +++ & + & 9.4 & 6 \\
\hline & & & & 172 & 228 & 97 & 150 & +++ & + & 8.5 & 6 \\
\hline
\end{tabular}

\section{References:}

1. Charak S Chikitsa Sthan,PRavidatta T, Chaukhamba S Sansthan, Varanasi, (2002) Reprint.

2. Prameha A, Sahasrayogam, Chaukhamba S Sansthan, Banaras Ayurveda Series 18, Dr. K. Nitheshwar.
3. Scheen AJ, Paquot N.(2013) Metformin revisited: A critical review of the benefit-risk balance in at-risk patients with type 2 diabetes. Diabetes Metab.;39:179-90.

4. Wickenberg J, Ingemansson S, Hlebowicz J. “(2010) Effects of Curcuma longa (turmeric) on postprandial plasma glucose and insulin in healthy subjects. Nutr J.; 9:43.
This work is licensed under Creative Commons Attribution 4.0 License

To Submit Your Article Click Here: Submit Article

DOI: $10.31579 / 2641-8975 / 017$
Ready to submit your research? Choose Auctores and benefit from:

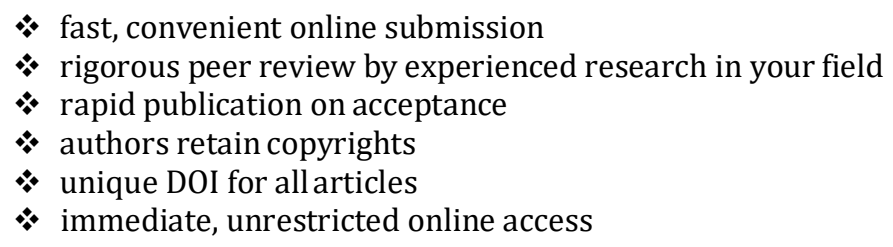

At Auctores, research is always in progress.

Learn more www.auctoresonline.org/journals/diabetes-and-islet-biology 